\title{
Aspectos epidemiológicos dos casos de sífilis em gestantes no Brasil de 2015 a 2020
}

Epidemiological aspects of syphilis cases in pregnant women in Brazil from 2015 to 2020

Aspectos epidemiológicos de los casos de sífilis en mujeres embarazadas en Brasil de 2015 a 2020

Suzana Cássia Feltrin Alves ${ }^{1 *}$, Ana Carolina Paiva Ferreira ${ }^{2}$, Ana Cláudia Cressoni De Conti ${ }^{3}$, Clara Melnick Fernandes ${ }^{4}$, Gabriella Carvalho Santos ${ }^{5}$, Giovanna Camarotto Patah ${ }^{5}$, Lara Stephanie Aparecida de Souza Jacob ${ }^{6}$, Letícia dos Reis Santos ${ }^{1}$, Mariah Marrara Cardozo ${ }^{7}$, Vanessa Munhoz Bitelman ${ }^{8}$.

\section{RESUMO}

Objetivo: Analisar a epidemiologia dos casos de sífilis em gestantes no Brasil no período de 2015 a 2020 .

Métodos: Estudo ecológico de corte transversal através da análise dos Indicadores e Dados Básicos da Sífilis nos Municípios Brasileiros do período de 2015 a 2020. Os dados foram coletados do Departamento de Doenças de Condições Crônicas e Infecções Sexualmente Transmissíveis (DCCI/SVS), disponibilizados através do Departamento de Informática do SUS, DATASUS. Foram incluídas todas as notificações realizadas no período supracitado, sendo excluídos os dados não computados pelo DATASUS. Os aspectos epidemiológicos analisados foram a faixa etária, raça, escolaridade e idade gestacional. Resultados: $\mathrm{A}$ análise dos dados permitiu concluir que o número de casos de infecções em gestantes ocorreu de forma crescente até $\mathrm{o}$ ano de 2017, tendo em 2018 discreta redução. O perfil epidemiológico predominante foi de gestantes com 20 e 29 anos, que se encontravam no primeiro trimestre da gravidez, da raça parda e que possuíam o Ensino Médio completo. Conclusão: $O$ estudo possibilitou determinar relações e variáveis que contribuíram para o crescente número de casos de sífilis gestacional no período avaliado, bem como a importância da disponibilização dos testes diagnósticos, ações de prevenção, orientações sobre saúde e disponibilização de tratamento.

Palavras-chave: Sífilis, Gravidez, Epidemiologia.

\footnotetext{
ABSTRACT

1 Universidade Estadual Paulista "Júlio de Mesquita Filho" (UNESP), Botucatu - SP.

*E-mail: suzana.feltrin@unesp.br

${ }^{2}$ Centro Universitário Maurício de Nassau (UNINASSAU), Recife - PE.

${ }^{3}$ Faculdade de Medicina de Catanduva (FAMECA), Catanduva - SP.

${ }^{4}$ Faculdade de Medicina do ABC (FMABC), Santo André - SP.

${ }^{5}$ Faculdade de Medicina de Jundiaí (FMJ), Jundiaí - SP.

6 Universidade Nove de Julho (UNINOVE), São Paulo - SP

7 Universidade Cidade de São Paulo (UNICID), São Paulo - SP.

8 Hospital Guilherme Álvaro, Boqueirão, Santos - SP
}

Objective: To analyze the epidemiology of syphilis cases in pregnant women in Brazil from 2015 to 2020. Methods: A cross-sectional ecological study through the analysis of Indicators and Basic Data of Syphilis in Brazilian Municipalities from 2015 to 2020. Data were collected from the Department of Diseases with Chronic Conditions and Sexually Transmitted Infections (DCCI/SVS), made available through the Department of Informatics of the SUS, DATASUS. All notifications made in the aforementioned period were included, excluding data not computed by DATASUS. The epidemiological aspects analyzed were age group, race, education and gestational age. Results: Data analysis allowed us to conclude that the number of cases of 
infections in pregnant women increased until the year 2017, with a slight reduction in 2018. The predominant epidemiological profile was of pregnant women aged between 20 and 29 years, who were in the first trimester of pregnancy, of mixed race and who had completed high school. Conclusion: The study made it possible to determine the relationships and variables that contributed to the growing number of cases of gestational syphilis in the period evaluated, as well as the importance of making diagnostic tests available, preventive actions, health guidelines and the availability of treatment.

Keywords: Syphilis, Pregnancy, Epidemiology.

\section{RESUMEN}

Objetivo: Analizar la epidemiología de los casos de sífilis en mujeres embarazadas en Brasil de 2015 a 2020. Métodos: Estudio ecológico transversal a través del análisis de Indicadores y Datos Básicos de Sífilis en Municipios brasileños de 2015 a 2020. Se analizaron los datos recolectados. del Departamento de Enfermedades Crónicas e Infecciones de Transmisión Sexual (DCCI/SVS), disponible a través del Departamento de Informática del SUS, DATASUS. Se incluyeron todas las notificaciones realizadas en el mencionado plazo, excluyendo los datos no computados por DATASUS. Los aspectos epidemiológicos analizados fueron grupo etario, raza, escolaridad y edad gestacional. Resultados: El análisis de los datos permitió concluir que el número de casos de infecciones en gestantes aumentó hasta el año 2017, con una leve reducción en el 2018. El perfil epidemiológico predominante fue el de gestantes con edad entre 20 y 29 años, que se encontraban en el primer trimestre del embarazo, de raza mestiza y con estudios secundarios completos. Conclusión: El estudio permitió determinar las relaciones y variables que contribuyeron al aumento del número de casos de sífilis gestacional en el período evaluado, así como la importancia de la disponibilidad de pruebas diagnósticas, acciones preventivas, lineamientos de salud y disponibilidad de tratamiento.

Palabras clave: Sífilis, Preñez, Epidemiología.

\section{INTRODUÇÃO}

A sífilis é uma Infecção Sexualmente Transmissível (IST) causada pela espiroqueta Treponema pallidum e representa um grave problema de saúde pública no Brasil. Segundo o levantamento da Organização Mundial da Saúde, a prevalência estimada desta patologia em gestantes foi de 0,69\% no ano de 2016, acometendo, anualmente, mais de 1 milhão de mulheres grávidas em todo o mundo (NUNES PS, et al., 2018; ROBERTS CP, et al., 2019; TORRES RG, et al., 2019).

A bactéria Treponema pallidum é um patógeno humano obrigatório pertencente à ordem Spirochaetales com elevada capacidade de invasão de tecidos e evasão do sistema imunológico, gerando processo inflamatório local não patognomônico, o qual pode mimetizar outras infecções. O curso da infecção ocorre tipicamente em estágios primário, secundário, latente e terciário, ao longo de um período de 10 ou mais anos. Na sífilis primária, as manifestações clínicas frequentes são a presença de úlcera única ou de inúmeras lesões em genitália, acompanhada de linfadenopatia regional. Após 6 a 8 semanas, a sífilis progride a um estágio secundário, marcado por sintomas como cefaleia, pirexia e erupções maculopapulares em diversas regiões da superfície corporal. Em sequência, uma longa fase de latência é frequente, podendo se estender por anos. A sífilis terciária, por sua vez, manifesta-se em indivíduos cuja infecção não foi tratada ou controlada. Nesta, são comuns acometimentos cardíacos, nervosos, ósseos e cutâneos importantes (PEELING RW, et al., 2017).

A transmissão acontece pelo contato sexual, com a entrada do T. pallidum na membrana mucosa genital. O risco de transmissão é maior durante as fases de sífilis primária e secundária, nas quais há maior carga da espiroqueta. Entretanto, a transmissão do agente pode ocorrer em qualquer período da gravidez (TSAI S, et al., 2019; TORRES RG, et al., 2019).

A transmissão também pode ocorrer de forma vertical para o feto in utero, e sem tratamento, há alta taxa de transmissão transplacentária. No caso de diagnóstico e tratamento precoces, este risco é reduzido em 
comparação com gestantes com intervenção medicamentosa tardia (ZHANG X, et al., 2018; HAWKES SJ, et al., 2013). Assim, o diagnóstico e o tratamento precoces reduzem as chances da ocorrência de transmissão vertical do agente infeccioso e previnem os possíveis riscos ao feto decorrentes desta, tais como a prematuridade, baixo peso ao nascer, aborto espontâneo tardio e óbito fetal (MAGALHÃES M, et al., 2017; NUNES PS, et al., 2018; WAN Z, et al., 2020).

Atualmente, o rastreamento da sífilis em mulheres grávidas possui caráter obrigatório, sendo realizado no primeiro e terceiro trimestres de idade gestacional. No entanto, apesar das medidas de prevenção e controle, há elevado subdiagnóstico, em especial nas regiões Norte e Nordeste, onde há maior restrição do acesso aos serviços de saúde (MACÊDO VC, et al., 2017; PEELING RW, et al., 2017). Nesse sentido, a realização inadequada do acompanhamento pré-natal se mostra diretamente relacionada a altos índices de sífilis congênita no Brasil (NUNES PS, et al., 2021).

Considerando a importância do tema, o presente estudo objetivou analisar os aspectos epidemiológicos da sífilis na gestação em mulheres brasileiras no período de 2015 a 2020, através das variáveis faixa etária, raça, escolaridade, classificação clínica da doença e idade gestacional.

\section{MÉTODOS}

Estudo epidemiológico de corte transversal através de análise dos casos de sífilis em gestantes disponíveis nos Indicadores e Dados Básicos da Sífilis nos Municípios Brasileiros do período de 2015 a 2020. Os dados foram coletados do Departamento de Doenças de Condições Crônicas e Infecções Sexualmente Transmissíveis (DCCI/SVS) e do Sistema de Informação de Agravos de Notificação (SINAN), disponibilizados pelo endereço eletrônico do Departamento de Informática do Sistema Único de Saúde (DATASUS).

O DCCI/SVS é o departamento do Ministério da Saúde responsável pelo monitoramento das infecções sexualmente transmissíveis e do HIV/Aids. O SINAN reúne e armazena notificações e investigações de casos de doenças e agravos registrados na lista nacional de notificação compulsória. O DATASUS possui como finalidade a coleta, processamento e divulgação de informações em saúde, possibilitando e disseminando o conhecimento epidemiológico no território nacional.

As variáveis analisadas foram faixa etária, raça, escolaridade e idade gestacional. A partir delas, o presente estudo buscou verificar em quais condições foram notificados maiores números de casos. Foram incluídas todas as notificações realizadas no período supracitado, sendo excluídos os dados não computados pelo DATASUS.

Os valores absolutos e a respectiva distribuição percentual de casos em cada variável foram reunidos e formatados em tabelas na ferramenta Microsoft Excel versão 2019, e posteriormente submetidos à análise estatística descritiva.

Uma vez que os dados armazenados pelo DATASUS estão disponíveis para acesso público, esta pesquisa não necessitou aprovação do Comitê de Ética em Pesquisa.

\section{RESULTADOS}

Diante da análise dos dados disponibilizados pelo DATASUS, foi possível determinar correlação entre a prevalência da sífilis gestacional e as variáveis idade materna, raça, escolaridade e idade gestacional.

Em relação ao número total de casos de sífilis gestacional, no período que compreendeu janeiro de 2015 a dezembro de 2020 , foram notificados 307.645 casos confirmados de sífilis em gestantes no país. Deste total, $63.250(20,56 \%)$ foram diagnosticados no ano de 2018, representando o maior número de notificações no período analisado. O menor, por sua vez, ocorreu no ano de 2015, com 32.778 (10,65\%) (Tabela 1).

Desta forma, de acordo com a análise quantitativa realizada, observou-se um aumento progressivo nas notificações entre 2015 a 2018. Por outro lado, uma expressiva diminuição pode ser notada nos últimos anos, período que compreende 2018 a 2020 (Tabela 1). 
Tabela 1 - Casos confirmados de sífilis em gestantes por ano de diagnóstico. Brasil, 2015-2020.

\begin{tabular}{llc}
\hline Variável & $\mathbf{N}$ & $\%$ \\
\hline Ano de diagnóstico & & 10,65 \\
\hline 2015 & 32778 & 12,45 \\
2016 & 38296 & 16,2 \\
2017 & 49835 & 20,56 \\
2018 & 63250 & 20,18 \\
2019 & 62084 & 19,96 \\
2020 & 61402 & 100 \\
\hline Total & 307645 & 100 \\
\hline
\end{tabular}

Fonte: Alves SCF, et al., 2022; dados extraídos do Sistema de Informação de Agravos de Notificação (SINAN), 2021.

Relativamente à idade gestacional, notou-se que o maior número de diagnósticos de sífilis ocorreu em mulheres que se encontravam no $1^{\circ}$ trimestre de gestação, equivalente a $38,5 \%$ dos casos totais, independente do ano analisado. Em segundo lugar, 29,4\% dos diagnósticos confirmados ocorreram no $3^{\circ}$ trimestre de idade gestacional, também em todos os períodos estudados (Tabela 2).

Tabela 2 - Casos confirmados de sífilis em gestantes e sua distribuição percentual segundo idade gestacional. Brasil, 2015-2020.

\begin{tabular}{|c|c|c|c|c|c|c|c|}
\hline Variável & 2015 & 2016 & 2017 & 2018 & 2019 & 2020 & Total \\
\hline \multicolumn{8}{|l|}{ Idade Gestacional } \\
\hline $1^{\circ}$ trimestre & 10563 & 14213 & 19815 & 24653 & 23997 & 25667 & 118908 \\
\hline $2^{\circ}$ trimestre & 9763 & 11011 & 13902 & 15884 & 14954 & 13441 & 78955 \\
\hline 30 trimestre & 10473 & 10759 & 13399 & 18762 & 18892 & 18473 & 90758 \\
\hline Idade gestacional ignorada & 1977 & 2238 & 2555 & 3662 & 4069 & 3751 & 18252 \\
\hline Ignorado & 4 & 75 & 165 & 289 & 174 & 1091 & 1798 \\
\hline Total & 32780 & 38296 & 49836 & 63250 & 62086 & 61441 & 308671 \\
\hline \multicolumn{8}{|l|}{ Distribuição percentual } \\
\hline $1^{\circ}$ trimestre & 32,2 & 37,1 & 39,8 & 39,0 & 38,7 & 41,8 & 38,5 \\
\hline $2^{\circ}$ trimestre & 29,8 & 28,8 & 27,9 & 25,1 & 24,1 & 21,9 & 25,6 \\
\hline 30 trimestre & 31,9 & 28,1 & 26,9 & 29,7 & 30,4 & 30,1 & 29,4 \\
\hline Idade gestacional ignorada & 6,0 & 5,8 & 5,1 & 5,8 & 6,6 & 6,1 & 5,9 \\
\hline Ignorado & 0,0 & 0,2 & 0,3 & 0,5 & 0,3 & 0,2 & 0,6 \\
\hline Total & 100 & 100 & 100 & 100 & 100 & 100 & 100 \\
\hline
\end{tabular}

Fonte: Alves SCF, et al., 2022; dados extraídos do Sistema de Informação de Agravos de Notificação (SINAN), 2021.

Ademais, ao longo dos anos estudados, os casos em todos os trimestres de idade gestacional sofreram elevação significativa, sobretudo no período de 2015 a 2018, fato que também pode ser observado nos casos 
classificados como "idade gestacional ignorada" e "ignorado" (Tabela 2). Também é importante ressaltar que, de 2018 a 2020, este padrão de aumento dos casos não se manteve, de forma que houve oscilações no número total de casos no $1^{\circ}$ e $3^{\circ}$ trimestres gestacionais e redução progressiva no $2^{\circ}$ trimestre gestacional.

No que concerne à idade das gestantes, em todas as faixas etárias analisadas, observou-se aumento significativo do número de casos de sífilis notificados entre os anos de 2015 e 2018. No entanto, entre 2018 e 2020, o aumento do número de casos só foi relatado nas mulheres com 20 a 29 anos, de forma que a proporção do aumento entre cada ano reduziu significativamente quando comparada aos anos anteriores. Por outro lado, em todas as demais faixas etárias, o número total de casos de sífilis gestacional notificados sofreu redução entre os anos de 2018 e 2020 (Tabela 3).

Tabela 3 - Casos confirmados de sífilis em gestantes e sua distribuição percentual segundo faixa etária. Brasil, 2015-2020

\begin{tabular}{cccccccc}
\hline Variável & $\mathbf{2 0 1 5}$ & $\mathbf{2 0 1 6}$ & $\mathbf{2 0 1 7}$ & $\mathbf{2 0 1 8}$ & $\mathbf{2 0 1 9}$ & $\mathbf{2 0 2 0}$ & Total \\
\hline Faixa etária & & & & & & & \\
\hline 10 a 14 anos & 452 & 519 & 624 & 718 & 644 & 620 & 3577 \\
15 a 19 anos & 8536 & 9942 & 12942 & 15742 & 14895 & 14329 & 76386 \\
20 a 29 anos & 16859 & 20086 & 26277 & 34058 & 34563 & 34636 & 166479 \\
30 a 39 anos & 6264 & 6988 & 8968 & 11505 & 10806 & 10650 & 55181 \\
40 ou mais & 669 & 740 & 985 & 1213 & 1167 & 1188 & 5962 \\
Ignorado & 0 & 1 & 6 & 5 & 0 & 2 & 14 \\
\hline Total & $\mathbf{3 2 7 8 0}$ & $\mathbf{3 8 2 7 6}$ & $\mathbf{4 9 8 0 2}$ & $\mathbf{6 3 2 4 1}$ & $\mathbf{6 2 0 7 5}$ & $\mathbf{6 1 4 2 5}$ & $\mathbf{3 0 7 5 9 9}$ \\
\hline Distribuição percentual & & & & & & & \\
\hline 10 a 14 anos & 1,4 & 1,4 & 1,3 & 1,1 & 1,0 & 1,0 & 1,2 \\
15 a 19 anos & 26,0 & 26,0 & 26,0 & 24,9 & 24,0 & 23,3 & 24,8 \\
20 a 29 anos & 51,4 & 52,5 & 52,8 & 53,9 & 55,7 & 56,4 & 54,1 \\
30 a 39 anos & 19,1 & 18,3 & 18,0 & 18,2 & 17,4 & 17,3 & 17,9 \\
40 ou mais & 2,0 & 1,9 & 2,0 & 1,9 & 1,9 & 1,9 & 1,9 \\
Ignorado & - & 0,0 & 0,0 & 0,0 & - & 0,0 & 0,0 \\
\hline Total & $\mathbf{1 0 0}$ & $\mathbf{1 0 0}$ & $\mathbf{1 0 0}$ & $\mathbf{1 0 0}$ & $\mathbf{1 0 0}$ & $\mathbf{1 0 0}$ & $\mathbf{1 0 0}$ \\
\hline
\end{tabular}

Fonte: Alves SCF, et al., 2022; dados extraídos do Sistema de Informação de Agravos de Notificação (SINAN), 2021.

Além disso, é notório que a maior incidência da infecção por Treponema pallidum em gestantes ocorre nas mulheres de 20 a 29 anos, correspondendo a mais da metade $(54,1 \%)$ de todos os casos de sífilis gestacional notificados. A segunda faixa etária com maior concentração de sífilis gestacional é a de 15 a 19 anos, seguida de 30 a 39 anos, totalizando $24,8 \%$ e 17,9\%, respectivamente, nos 6 anos analisados (Tabela 3).

Em relação ao grau de escolaridade, em mulheres com $1^{\mathrm{a}}$ à $4^{\mathrm{a}}$ série completa, $5^{\mathrm{a}}$ a $8^{\mathrm{a}}$ série incompleta ou Ensino fundamental completo, o número total de casos de sífilis gestacional aumentou a cada ano entre 2015 e 2018, passando a reduzir anualmente entre 2018 e 2020, o que também pode ser observado no grupo de mulheres com escolaridade ignorada, o qual concentrou a maior quantidade de casos notificados no período estudado (26,9\%) (Tabela 4). 
Tabela 4 - Casos confirmados de sífilis em gestantes e sua distribuição percentual segundo escolaridade. Brasil, 2015-2020.

\begin{tabular}{|c|c|c|c|c|c|c|c|}
\hline Variável & 2015 & 2016 & 2017 & 2018 & 2019 & 2020 & Total \\
\hline \multicolumn{8}{|l|}{ Escolaridade } \\
\hline Analfabeto & 205 & 245 & 529 & 493 & 238 & 165 & 1875 \\
\hline $1^{\mathrm{a}}$ à $4^{\mathrm{a}}$ série incompleta & 1887 & 1911 & 2106 & 2534 & 2074 & 1926 & 12438 \\
\hline $4^{a}$ série incompleta & 1296 & 1418 & 1702 & 1938 & 1913 & 1799 & 10066 \\
\hline $5^{\mathrm{a}}$ à $8^{\mathrm{a}}$ série incompleta & 6509 & 7711 & 9705 & 11890 & 10901 & 10243 & 56959 \\
\hline Ensino fund. completo & 3182 & 3709 & 4983 & 6309 & 6150 & 5981 & 30314 \\
\hline Ensino médio incompleto & 4568 & 5548 & 7507 & 9385 & 9517 & 9541 & 46066 \\
\hline Ensino médio completo & 5012 & 6207 & 9053 & 12224 & 12920 & 13774 & 59190 \\
\hline Superior incompleto & 391 & 467 & 745 & 916 & 948 & 967 & 4434 \\
\hline Superior completo & 257 & 352 & 541 & 722 & 768 & 833 & 3473 \\
\hline Não se aplica & 0 & 20 & 19 & 15 & 21 & 36 & 111 \\
\hline Ignorado & 9473 & 10708 & 12946 & 16824 & 16636 & 16176 & 82763 \\
\hline Total & 32780 & 38296 & 49836 & 63250 & 62086 & 61441 & 307689 \\
\hline \multicolumn{8}{|l|}{ Distribuição percentual } \\
\hline Analfabeto & 0,6 & 0,6 & 1,1 & 0,8 & 0,4 & 0,3 & 0,6 \\
\hline $1^{\mathrm{a}}$ à $4^{\mathrm{a}}$ série incompleta & 5,8 & 5,0 & 4,2 & 4,0 & 3,3 & 3,1 & 4,0 \\
\hline $4^{\mathrm{a}}$ série completa & 4,0 & 3,7 & 3,4 & 3,1 & 3,1 & 2,9 & 3,3 \\
\hline $5^{\mathrm{a}}$ à $8^{\mathrm{a}}$ série incompleta & 19,9 & 20,1 & 19,5 & 18,8 & 17,6 & 16,7 & 18,5 \\
\hline Ensino fund. completo & 9,7 & 9,7 & 10,0 & 10,0 & 9,9 & 9,7 & 9,9 \\
\hline Ensino médio incompleto & 13,9 & 14,5 & 15,1 & 14,8 & 15,3 & 15,5 & 15 \\
\hline Ensino médio completo & 15,3 & 16,2 & 18,2 & 19,3 & 20,8 & 22,4 & 19,2 \\
\hline Superior incompleto & 1,2 & 1,2 & 1,5 & 1,4 & 1,5 & 1,6 & 1,4 \\
\hline Superior completo & 0,8 & 0,9 & 1,1 & 1,1 & 1,2 & 1,4 & 1,1 \\
\hline Não se aplica & - & 0,1 & 0,0 & 0,0 & 0,0 & 0,1 & 0,0 \\
\hline Ignorado & 28,9 & 28,0 & 26,0 & 26,6 & 26,8 & 26,3 & 26,9 \\
\hline Total & 100 & 100 & 100 & 100 & 100 & 100 & 100 \\
\hline
\end{tabular}

Fonte: Alves SCF, et al., 2022; dados extraídos do Sistema de Informação de Agravos de Notificação (SINAN), 2021.

Ainda nesta variante, vale destacar o grupo de mulheres analfabetas, que também apresentaram um comportamento de aumento seguido de redução, porém esta diminuição iniciou-se um ano antes em relação aos grupos citados anteriormente. Além disso, as mulheres analfabetas concentraram os menores índices de infecção por Treponema pallidum em todos os anos entre 2015 e 2020, totalizando 0,6\% do total de casos deste período (Tabela 4).

Nos demais grupos de mulheres de acordo com a escolaridade, Ensino Médio incompleto, Ensino Médio completo, Superior completo e Superior incompleto, o aumento dos casos notificados ocorreu em todos os anos entre 2015 e 2020, sem nenhum período de redução (Tabela 4). 
Por fim, atrás do grupo de mulheres com grau de escolaridade ignorado, as maiores quantidades de casos notificados de sífilis gestacional ocorreram em mulheres com Ensino Médio completo $(19,2 \%)$ e $5^{\mathrm{a}}$ a $8^{\mathrm{a}}$ série incompleta (18,5\%), respectivamente (Tabela 4).

Outra variável analisada no presente estudo epidemiológico foi a raça das gestantes. Conforme dados do SINAN, é notório que as mulheres pertencentes à raça parda constituem o grupo mais acometido pela doença nos últimos anos, compreendendo $50 \%$ do total dos casos ao longo dos seis anos estudados (2015 a 2020), seguido das mulheres brancas, com $29,2 \%$ dos casos totais.

Vale destacar também as mulheres indígenas e amarelas, que representam as raças menos acometidas pela infecção do Treponema pallidum, com um total de $0,5 \%$ e $0,9 \%$, respectivamente, não ultrapassando a taxa de $1 \%$ dentre as infectadas em nenhum dos anos analisados (Tabela 5).

Tabela 5 - Casos confirmados de sífilis em gestantes e sua distribuição percentual segundo raça/cor. Brasil, 2015-2020.

\begin{tabular}{cccccccc}
\hline Variável & $\mathbf{2 0 1 5}$ & $\mathbf{2 0 1 6}$ & $\mathbf{2 0 1 7}$ & $\mathbf{2 0 1 8}$ & $\mathbf{2 0 1 9}$ & $\mathbf{2 0 2 0}$ & Total \\
\hline Raça/cor & & & & & & & \\
\hline Branca & 10120 & 11803 & 15291 & 18121 & 17744 & 16841 & 89920 \\
Preta & 3976 & 4789 & 6321 & 7715 & 7347 & 7392 & 37540 \\
Amarela & 262 & 323 & 459 & 605 & 590 & 643 & 2882 \\
Parda & 15310 & 18044 & 24210 & 32085 & 31785 & 32384 & 153818 \\
Indígena & 225 & 228 & 266 & 331 & 312 & 292 & 1654 \\
Ignorado & 2887 & 3109 & 3289 & 4393 & 4308 & 3889 & 21875 \\
\hline Total I & $\mathbf{3 2 7 8 0}$ & $\mathbf{3 8 2 9 6}$ & $\mathbf{4 9 8 3 6}$ & $\mathbf{6 3 2 5 0}$ & $\mathbf{6 2 0 8 6}$ & $\mathbf{6 1 4 4 1}$ & $\mathbf{3 0 7 6 8 9}$ \\
\hline Distribuição percentual & & & & & & & \\
\hline Branca & 30,9 & 30,8 & 30,7 & 28,6 & 28,6 & 27,4 & 29,2 \\
Preta & 12,1 & 12,5 & 12,7 & 12,2 & 11,8 & 12,0 & 12,2 \\
Amarela & 0,8 & 0,8 & 0,9 & 1,0 & 1,0 & 1,0 & 0,9 \\
Parda & 46,7 & 47,1 & 48,6 & 50,7 & 51,2 & 52,7 & 50,0 \\
Indígena & 0,7 & 0,6 & 0,5 & 0,5 & 0,5 & 0,5 & 0,5 \\
Ignorado & 8,8 & 8,1 & 6,6 & 6,9 & 6,9 & 6,3 & 7,1 \\
\hline Total & $\mathbf{1 0 0}$ & $\mathbf{1 0 0}$ & $\mathbf{1 0 0}$ & $\mathbf{1 0 0}$ & $\mathbf{1 0 0}$ & $\mathbf{1 0 0}$ & $\mathbf{1 0 0}$
\end{tabular}

Fonte: Alves SCF, et al., 2022; dados extraídos do Sistema de Informação de Agravos de Notificação (SINAN), 2021.

Ainda, é importante salientar que não houve um padrão de evolução da quantidade de casos comum às raças durante a progressão dos seis anos, de forma que em alguns grupos as taxas aumentaram, em outros, reduziram e em outros, variaram (Tabela 5).

Finalmente, é possível determinar o padrão de distribuição dos tipos de sífilis que acometem as gestantes no Brasil, analisando a quantidade dos casos de sífilis gestacional, de acordo com suas classificações clínicas.

Observando a porcentagem total de casos de sífilis gestacional, a forma latente da infecção foi a mais prevalente, a qual somou $33,7 \%$ em todo o período estudado (2015 a 2020), seguida da sífilis primária (27\% no total). Porém, cabe ressaltar que em 2015 e 2016 a quantidade de sífilis primária era maior que a 
quantidade de sífilis latente e que as posições se inverteram de 2017 em diante, quando a forma latente passou a ser a mais comum entre as gestantes (Tabela 6). Em relação às formas latente e primária, as sífilis secundária e terciária apresentam uma prevalência bem menor (5\% e 9,6\%, respectivamente) e ambas tiveram uma redução na porcentagem de casos, quando se compara o início (2015) e o final (2020) do período analisado (Tabela 6).

Tabela 6 - Casos confirmados de sífilis em gestantes e sua distribuição percentual segundo classificação clínica. Brasil, 2015-2020.

\begin{tabular}{cccccccc}
\hline Variável & $\mathbf{2 0 1 5}$ & $\mathbf{2 0 1 6}$ & $\mathbf{2 0 1 7}$ & $\mathbf{2 0 1 8}$ & $\mathbf{2 0 1 9}$ & $\mathbf{2 0 2 0}$ & Total \\
\hline \multicolumn{1}{c}{ Classificação clínica } & & & & & & & \\
\hline Sífilis primária & 10103 & 11155 & 14099 & 16740 & 15485 & 15408 & 82990 \\
Sífilis secundária & 1901 & 2159 & 2620 & 3199 & 3000 & 2550 & 15429 \\
Sífilis terciária & 3501 & 4115 & 5389 & 6115 & 5149 & 5376 & 29645 \\
Sífilis latente & 8094 & 10628 & 15196 & 21650 & 23657 & 24440 & 103665 \\
$\quad$ Ignorado & 9181 & 10239 & 12532 & 15546 & 14795 & 13667 & 95960 \\
\hline Total & $\mathbf{3 2 7 8 0}$ & $\mathbf{3 8 2 9 6}$ & $\mathbf{4 9 8 3 6}$ & $\mathbf{6 3 2 5 0}$ & $\mathbf{6 2 0 8 6}$ & $\mathbf{6 1 4 4 1}$ & $\mathbf{3 0 7 6 8 9}$ \\
\hline Distribuição percentual & & & & & & & \\
\hline$\quad$ Sífilis primária & 30,8 & 29,1 & 28,3 & 26,5 & 24,9 & 25,1 & 27,0 \\
$\quad$ Sífilis secundária & 5,8 & 5,6 & 5,3 & 5,1 & 4,8 & 4,2 & 5,0 \\
$\quad$ Sífilis terciária & 10,7 & 10,7 & 10,8 & 9,7 & 8,3 & 8,7 & 9,6 \\
$\quad$ Sífilis latente & 24,7 & 27,8 & 30,5 & 34,2 & 38,1 & 39,8 & 33,7 \\
$\quad$ Ignorado & 28,0 & 26,7 & 25,1 & 24,6 & 23,8 & 22,2 & 24,7 \\
\hline Total & $\mathbf{1 0 0}$ & $\mathbf{1 0 0}$ & $\mathbf{1 0 0}$ & $\mathbf{1 0 0}$ & $\mathbf{1 0 0}$ & $\mathbf{1 0 0}$ & $\mathbf{1 0 0}$
\end{tabular}

Fonte: Alves SCF, et al., 2022; dados extraídos do Sistema de Informação de Agravos de Notificação (SINAN), 2021.

\section{DISCUSSÃO}

De acordo com os resultados disponibilizados pelo SINAN, houve um aumento progressivo das notificações de casos confirmados de sífilis em gestantes no período de 2015 a 2018. Esse aumento pode ser explicado pela literatura pela maior utilização de testes rápidos por gestantes, garantindo maior cobertura diagnóstica, e, também, por mudanças no comportamento das mulheres e seus parceiros nas relações sexuais ao absterem-se do uso de preservativos.

Vale ressaltar, também, que o aumento do número de gestantes com sífilis pode ser justificado a partir da ineficácia das ações de prevenção da doença. Além disso, a carência de penicilina, fármaco de escolha para o tratamento da infecção, em unidades de atendimento de localidades socioeconomicamente menos favorecidas pode estar relacionada à prevalência e manutenção da sífilis gestacional (GODOI NETO L, et al., 2019).

A esse respeito, Marques JVS, et al. (2018), apontou a falta do medicamento em $60 \%$ dos estados brasileiros em 2016. A falta de matéria prima para a produção do medicamento em âmbito global pode ter ocasionado tal escassez (MOROSKOSKI M, et al., 2018).

Em um estudo transversal realizado no estado de Minas Gerais, foi observado que a maior atenção às gestantes na assistência pré-natal evidenciou os casos da patologia nessa população. Entretanto, acreditase que os casos de sífilis em gestantes apresentam-se subnotificados, tendo havido, portanto, um maior 
contingente de mulheres grávidas com a doença no período estudado (ANDRADE HS, et al., 2019; MARQUES JVS, et al., 2018). Outro estudo abordou que a melhora nas ações de vigilância epidemiológica nos últimos anos acarretou a diminuição dos sub-registros da infecção (SOUZA BSO, et al., 2018).

Em relação ao período compreendido pelos anos de 2018 a 2020, observou-se discreta redução do número de casos confirmados de sífilis gestacional. Uma das possíveis justificativas para esse fato foi a implantação, em 2018, do Projeto de Resposta Rápida à Sífilis nas Redes de Atenção pelo Ministério da Saúde, visando diminuir a sífilis adquirida, congênita e gestacional (OLIVEIRA JÚNIOR SA, et al., 2021).

Quanto à idade gestacional, foi possível constatar que os diagnósticos confirmados da patologia se concentraram no $1^{\circ}$ trimestre de gestação, seguido do $3^{\circ}$ trimestre, correspondendo aos períodos da gestação nos quais a triagem da sífilis por meio de testes sorológicos é obrigatória (OLIVEIRA IM, et al., 2021). Não obstante, Lafetá KRG, et al. (2016) observou em uma pesquisa retrospectiva em serviço de referência em infectologia da cidade de Montes Claros - MG, que a maioria das gestantes incluídas no estudo foram diagnosticadas no ato do parto ou na curetagem.

Nesse sentido, tal predominância pode ser explicada pela busca tardia pelo pré-natal ou a descontinuidade do acompanhamento no decorrer da gestação, além da baixa efetividade desta assistência, quando existente. Desta forma, evidencia-se que o diagnóstico e o tratamento da sífilis na gestação, pelos testes e condutas preconizados pelo Ministério da Saúde devem ser oportunamente realizados desde as primeiras semanas de gravidez, a fim de se evitar desfechos negativos ao feto (CAVALCANTE PAM, et al., 2017; FURTADO MFS, et al., 2017).

A respeito da faixa etária, a compreendida entre os 20 e 29 anos foi a mais acometida. Este achado é compartilhado por outros estudos anteriores, e se relaciona ao fato de que o desempenho sexual das mulheres geralmente é mais evidente neste período da vida reprodutiva (ANDRADE HS, et al., 2019; ROEHRS MP, et al., 2020; SOUZA BSO, et al., 2018).

Considerando também a faixa etária de adolescentes e jovens (10 a 19 anos), observou-se um aumento no número de casos confirmados. Moroskoski M, et al. (2018) e Barbosa DRM, et al. (2017) obtiveram resultados semelhantes, nos quais gestantes com 15 a 19 anos representaram a maior parcela de grávidas adolescentes. Estes dados podem ser elucidados pela concepção de que, no decorrer dos últimos anos, a vida sexual de meninas e meninos tem se iniciado mais precocemente, muitas vezes de maneira desprotegida (SOUZA BSO, et al., 2018).

Ademais, a crescente de diagnósticos em adolescentes demonstra a falta de aderência destes às ações de conscientização em torno das IST havendo a utilização esporádica de preservativos durante as relações sexuais. Dentre os motivos do ineficiente emprego desta proteção, Moroskoski M, et al. (2018), citando Jardim DP e Santos EF (2012), elencou que, de acordo com a percepção dos jovens, o método interfere no prazer e seu uso é desnecessário, uma vez que estivessem sob uso de pílula anticoncepcional e que confiassem em seus parceiros.

Deve-se ressaltar, ainda, que o uso de entorpecentes como drogas ilícitas ou psicoativas e álcool, pode aumentar o risco da contração de sífilis em gestantes jovens, em especial quando estas se encontram em situação de rua (MACÊDO VC, et al., 2017; MOROSKOSKI M, et al., 2018).

Outra variável estudada foi a escolaridade e sua relação com o diagnóstico de sífilis em gestantes. Assim, os índices em mulheres com ensino médio completo predominaram em relação aos demais, seguida daquelas com $5^{\mathrm{a}}$ a $8^{\mathrm{a}}$ série incompleta. Os menores percentuais encontrados foram os de mulheres analfabetas e com ensino superior completo.

Souza BSO, et al. (2018) ressaltaram em seu estudo que a baixa escolaridade se destaca como fator de exposição da sífilis entre as gestantes, uma vez que, nesse contexto, há pouco ou nenhum conhecimento acerca dos métodos de prevenção de infecções sexualmente transmissíveis, expondo a mãe e o recémnascido a potenciais riscos, tais quais o baixo peso da criança ao nascer e a mortalidade infantil (CABRAL BTV, et al., 2018). 
Além disso, Andrade HS, et al. (2019), em seu estudo acerca da epidemiologia da sífilis no sexo feminino, discorreu que a maior proporção de casos da doença em baixa escolaridade pode decorrer do fato de que as mulheres se dedicavam mais ao trabalho do que aos estudos, compondo uma vertente socioeconômica importante. Entende-se, portanto, que mulheres em condições de renda e qualidade de assistência à saúde desfavorecidas, estão mais suscetíveis à infecção pelo $T$. pallidum, apesar de que a doença pode se apresentar em qualquer nível social.

Assim como o encontrado em estudos semelhantes, a raça/cor parda foi aquela que somou maior número de casos confirmados no período em questão e, em segundo lugar, a raça/cor branca. Moroskoski M, et al. (2018) destacou, tal como demais pesquisas acerca do assunto, que indivíduos pardos e negros estão sujeitos a menores níveis de escolaridade e a residirem em localidades menos favorecidas em termos de infraestrutura e qualidade de vida.

O mesmo estudo constatou que, de acordo com os indicadores de acesso à saúde do Ministério da Saúde, a adesão ao acompanhamento pré-natal é maior em gestantes autodeclaradas brancas, enquanto em pretas e pardas, houve expressiva diminuição deste índice. Logo, fica evidente que o acesso ao atendimento médico é menor nessa população, emergindo a necessidade da implementação de ações em saúde pública para corrigir este desfalque (MOROSKOSKI M, et al., 2018).

A variável classificação clínica, por sua vez, apresentou percentuais bastantes semelhantes entre as classificações sífilis primária, sífilis latente e classificação ignorada. Ainda assim, a maioria dos casos foram diagnosticados no estágio de sífilis latente. No entanto, Cavalcante PAM, et al. (2017), em estudo realizado no estado do Tocantins, demonstraram que a classificação da sífilis em gestantes foi sobremaneira equivocada, visto que, apesar de a fase latente ser a mais incidente nos dados do SINAN, foi constatado um alto percentual de gestantes em fase primária e terciária da infecção. Resultados correlatos foram observados no estado de Goiás (OLIVEIRA IM, et al., 2021).

Por outro lado, nos estudos de Souza BSO, et al. (2018) e de Marques JVS, et al. (2018), tais dados foram divergentes: nestes, a sífilis terciária e classificação ignorada obtiveram os maiores índices de diagnóstico na população gestante (OLIVEIRA IM, et al., 2021).

Estes erros de classificação do estágio da doença são explicados pela literatura pela dificuldade em se realizar o diagnóstico, seja pela manifestação assintomática do cancro duro, seja pela restrita visibilidade das lesões, quando presentes, devido à sua localização. É importante ressaltar que a ficha utilizada para a notificação de casos de sífilis em gestantes não permite a diferenciação da sífilis latente em recente e tardia, acarretando em condutas terapêuticas inadequadas e inespecíficas (CAVALCANTE PAM, et al., 2017; MOROSKOSKI M, et al., 2018; SOUZA BSO, et al., 2018).

No tocante aos parceiros sexuais das gestantes diagnosticadas, a bibliografia consultada estabeleceu que a negligência em relação ao uso de preservativos é mais presente em relações estáveis e casamentos, nas quais a busca por melhor desempenho sexual e a segurança no parceiro distanciam o conjunto da proteção contra infecções sexualmente transmissíveis (ROEHRS MP, et al., 2020).

Pesquisas neste contexto abordaram, também, que a maioria dos parceiros sintomáticos sequer recebeu tratamento para a sífilis, mostrando-se mais evidente a necessidade de que a terapia concomitante de ambos seja realizada para que sejam obtidos resultados clínicos efetivos (BARBOSA DRM, et al., 2017; SOUZA BSO, et al., 2018).

Por fim, convém ressaltar que o presente trabalho se refere a dados coletados em registros de fontes oficiais, limitando a análise do perfil epidemiológico das gestantes aos resultados expostos pelo SINAN. Desta forma, não foi possível se considerar possíveis sub-registros e erros inerentes ao cômputo das notificações, tais quais erros de classificação ou preenchimento (BARBOSA DRM, et al., 2017).

\section{CONCLUSÃO}

De forma geral, o aumento na notificação dos casos ao longo do período entre 2015 a 2020 se deve à maior cobertura diagnóstica decorrente da ampliação do uso de testes rápidos para detecção da infecção, e também decorre da carência de alguns setores da saúde na prevenção e combate à doença. Analisando as 
variáveis individualmente, conclui-se que os casos de sífilis gestacional são mais prevalentes em mulheres na faixa etária de 20 e 29 anos, sobretudo nas que se encontram no primeiro trimestre da gravidez. Quanto à raça, a predominância da infecção por Treponema pallidum ocorre em gestantes pardas e, por fim, relativamente à escolaridade, a maioria dos casos de sífilis gestacional se concentra em mulheres que possuem o Ensino Médio completo. Em suma, o estudo em questão possibilitou determinar relações e variáveis que contribuíram para o crescente número de casos de sífilis gestacional no período avaliado, bem como a importância da disponibilização dos testes diagnósticos, ações de prevenção, orientações sobre saúde e disponibilização de tratamento.

\section{REFERÊNCIAS}

1. ANDRADE HS, et al. Caracterização epidemiológica dos casos de sífilis em mulheres. Ciência \& Saúde, 2019; 12(1): 32124.

2. BARBOSA DRM, et al. Perfil epidemiológico dos casos de sífilis gestacional. Revista de enfermagem UFPE online, 2017; 11(5): 1867.

3. CABRAL BTV, et al. Sífilis em gestante e sífilis congênita: Um estudo retrospectivo. Revista Ciência Plural, 2018; 3(3): 32-44.

4. CAVALCANTE PAM, et al. Sífilis gestacional e congênita em Palmas, Tocantins, 2007-2014. Epidemiologia e serviços de saúde: Revista do Sistema Único de Saúde do Brasil, 2017; 26(2): 255-264.

5. DOMINGUES RMSM, et al. Prevalência de sífilis e HIV em gestantes encarceradas e incidência de sífilis congênita em crianças nascidas em prisões brasileiras. Cadernos de Saúde Pública, 2017; 33(11): 1-15.

6. FURTADO MFS, et al. Fatores epidemiológicos da sífilis em gestantes no município de São Luís-MA. Revista Uningá, 2017; 52(1): 51-55.

7. GODOI NETO L, et al. Epidemiologia da sífilis gestacional e congênita no estado de Goiás no período de 2013 a 2018. Revista Brasileira Militar de Ciências, 2019; 5(13): 13-20.

8. HAWKES SJ, et al. Early antenatal care: Does it make a difference to outcomes of pregnancy associated with syphilis? A systematic review and meta-analysis. PLoS ONE, 2013; 8(2): 1-7.

9. JARDIM DP, SANTOS EF. Uso do preservativo masculino por adolescentes no início da vida sexual. Adolescência e Saúde, 2012; 9(2): 37-44.

10. LAFETÁ KRG, et al. Sífilis materna e congênita, subnotificação e difícil controle. Revista Brasileira de Epidemiologia, $2016 ; 19(1): 63-74$.

11. MACÊDO VC, et al. Risk factors for syphilis in women: case-control study. Revista de Saúde Pública, 2017; 51(78): 112.

12. MAGALHÃES M, et al. Sífilis na gravidez e sífilis congênita: Realidade de um hospital universitário central português. Revista Brasileira de Ginecologia e Obstetrícia, 2017; 39(6): 265-272.

13. MOROSKOSKI M, et al. Perfil de gestantes adolescentes diagnosticadas com sífilis em Curitiba-PR. Revista de Saúde Pública do Paraná, 2018; 1(1): 47-58.

14. NUNES PS, et al. Sífilis gestacional e congênita e sua relação com a cobertura da Estratégia Saúde da Família, Goiás, 2007-2014: um estudo ecológico. Epidemiologia e serviços de saúde: Revista do Sistema Único de Saúde do Brasil, 2018; 27(4): e2018127.

15. NUNES PS, et al. Tendência temporal e distribuição espacial da sífilis gestacional e congênita em Goiás, 2007-2017: um estudo ecológico. Epidemiologia e serviços de saúde: revista do Sistema Único de Saúde do Brasil, 2021; 30(1): e2019371.

16. OLIVEIRA IM, et al. Diagnóstico, tratamento e notificação da sífilis durante a gestação em Goiás, de 2007 a 2017. Revista de Saúde Pública, 2021; 55: 68.

17. OLIVEIRA JUNIOR SA, et al. Perfil epidemiológico da Sífilis gestacional e sua influência na transmissão vertical no Município de Parnamirim-RN. Research, Society and Development, 2021; 10(10): 1-10.

18. PEELING RW, et al. Syphilis. Nature Reviews Disease Primers, 2017; 3(17073): 1-21.

19. ROBERTS CP, et al. Alternative treatments for syphilis during pregnancy. Sexually Transmitted Diseases, 2019 ; 46(10): 637-640.

20. ROEHRS MP, et al. Sífilis materna no Sul do Brasil: epidemiologia e estratégias para melhorar. Femina, 2020; 48(12): 753-759.

21. MARQUES JVS, et al. Perfil Epidemiológico da sífilis gestacional: Clínica e evolução de 2012 a 2017. Revista de Políticas Públicas, 2018; 17(2): 13-20.

22. SOUZA BSO, et al. Análise epidemiológica de casos notificados de sífilis. Revista da Sociedade Brasileira de Clínica Médica, 2018; 16(2): 94-98.

23. TORRES RG, et al. Syphilis in pregnancy: The reality in a public hospital. Revista Brasileira de Ginecologia e Obstetrícia, 2019; 41(2): 90-96.

24. TSAI S, et al. Syphilis in pregnancy. Obstetrical and Gynecological Survey, 2019; 74(9): 557-564.

25. WAN Z, et al. Maternal syphilis treatment and pregnancy outcomes: a retrospective study in Jiangxi Province, China. BMC Pregnancy and Childbirth, 2020; 20(1): 1-8.

26. ZHANG X, et al. Surveillance of maternal syphilis in China: Pregnancy outcomes and determinants of congenital syphilis. Medical Science Monitor, 2018; 24: 7727-7735. 